\title{
Contractile and Histochemical Properties of Sliced Muscle Grafts Regenerating in Normal and Denervated Rat Limbs
}

\author{
Bruce M. Carlson ${ }^{1}$ and Ernest Gutmann \\ Department of Anatomy, University of Michigan, Ann Arbor, Michigan 48104; and \\ Institute of Physiology, ¿SAV, Budéjovická 1083, Praha, KRC, Cachoslovakia
}

Received August 15, 1975

Rat extensor digitorum longus muscles were transversely sliced into 7-8 segments. The muscle slices were autografted back into their original beds. In one series the recipient limbs were normal and in the olher, limbs were denervated. At postoperative intervals of $7,14,30$, and 60 days, the contractile properties (latency period, contraction and half relaxation times, time parameters of contraction of twitch and tetanus, and twitch and tetanic tension) and histochemical properties (succinic dehydrogenase and myofibrillar ATPase) were analyzed. Sliced grafts regenerating in normally innervated legs followed a typical conversion from slow to fast contraction times, whereas regenerates in denervated limbs remained slow. Histochemically, innervated regenerates developed a heterogeneous pattern of muscle fiber type staining during the second month, whereas different histochemical types of muscle fibers did not appear in noninnervated regenerates. As in ontogeny, denervation retards or prevents the full structural and functional differentiation of regenerating muscle fibers.

\section{INTRODUCTION}

It is now well established that mammalian skeletal muscle possesses a substantial regenerative capacity and that regenerated muscle fibers achieve a high degree of contractility in response to direct stimulation or indirect stimulation through the nerve $(3,17)$. In the development of contractile properties, a regenerating muscle recapitulates the ontogenetic pattern, i.e., a regenerating fast muscle at first contracts very slowly but progressively speeds up until almost normal speeds of contraction are reached (3).

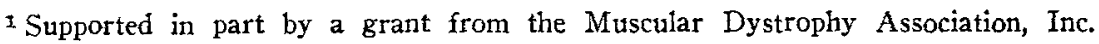
This research was carried out during a scientific exchange visit sponsored by the Academies of Sciences of Czechoslovakia and the United States. 
One of the persistent questions facing those studying both the morphology and the function of regenerating muscle is the extent to which regeneration can proceed in the absence of innervation. In one of the early statements on this question, Studitsky et al. (19) maintained that regeneration does not continue past the myoblast stage. Zhenevskaya (20), however, noted the formation of myotubes in denervated minced nnuscle regenerates of the rat, but found that the further differentiation of muscle fibers was defective although some striated muscle fibers did appear. In the denervated limb of the frog, Hsu (10) found large numbers of regenerating myotubes, but soon thereafter a massive degenerative response set in, leaving at most only a few striated muscle fibers by the end of a month.

The ability of rat muscle to regenerate in the absence of nerves has been recently reinvestigated in two different experimental systems. Mong (11, 12) has studied the regenerative ability of the minced gastrocnemius muscle of the rat in the absence of nerves with histochemical and quantitative morphological techniques. He found virtually no consistent differences between minced muscle regeneration in normal and in denervated limbs during the first postoperative month. Later, while the innervated muscle fibers underwent rapid growth and histochemical differentiation into distinct fiber types, the muscle fibers in noninnervated regenerates began a period of gradual atrophy and did not form histochemically distinguishable fiber types. In the work reported here the free grafting model of muscle regeneration was used, and our attention was focused primarily upon the development of contractile and histochemical properties of regenerating muscle fibers found in free grafts within denervated limbs. In order to eliminate the "contamination" by surviving original muscle fibers, which is the rule in free grafts $(4,5)$, the recently developed model of grafting sliced muscles was used (8). This way a pure population of regenerating muscle fibers could be obtained.

\section{MATERIALS AND METHODS}

For these experiments, 58 one-month-old Wistar rats were used. Under ether anesthesia the extensor digitorum longus muscle was completely removed from the leg and transversely sliced into 7-8 segments. These slices were then replaced into the muscle bed in as nearly normal orientation as possible. The muscle slices were held in place by suturing the overlying fascial sheath and the skin. At the same time as the muscle slicing operation, the leg was denervated by section and ligation of the sciatic nerve high up in the thigh. For control purposes, sliced extensor digitorum longus muscles were also grafted into a series of normally innervated limbs. In these animals, innervation of the grafts was obtained by placing the cut end of the motor nerve to the extensor digitorum longus alongside the 
reimplanted muscle slices. Muscles regenerating in normally innervated limbs were examined $7,14,30$, and 60 days postoperatively whereas those in denervated limbs were analyzed at 14,30 , and 60 days. One group of normal extensor digitorum longus muscles from 8 animals of the same age as the experimentals was analyzed to provide a background of normal control data.

At the time of analysis the rats were anesthetized with ether, and the regenerated muscles were quickly removed. They were immediately placed in an oxygenated solution containing $149.8 \mathrm{~mm} \mathrm{Na}^{+}, 5.0 \mathrm{~mm} \mathrm{~K}, 2.0 \mathrm{~mm}$ $\mathrm{Ca}^{2+}, 148.0 \mathrm{~mm} \mathrm{Cl}-12.0 \mathrm{~mm} \mathrm{HCO}_{3}^{-}, 1.0 \mathrm{mM} \mathrm{H}_{2} \mathrm{PO}_{4}^{-}$, and $11.0 \mathrm{~mm}$ glucose at $\mathrm{pH}$ 7.2. The regenerates were equilibrated in this solution for 10 min in the presence of $0.01 \mathrm{M}$ tubocurarine chloride (Merck) and then prepared for massive direct stimulation as described earlier (3). After determination of the optimal resting tension for isometric recording, contractile properties of twitch and tetanus were recorded by an automatic analyzer of muscle contraction properties (15). The following parameters were measured: twitch and tetanic tension, latency period (stimulus to first mechanical response), contraction time (time to peak), half relaxation time (from peak to half amplitude of the spike) and the maximal rate of tension development (or time parameter of contraction) for both twitch and tetanus. The time parameter of contraction has been defined (16) as the time necessary for reaching maximal force of contraction, assuming that the contraction takes place from zero to maximum at a linear rate equal to the maximal rate of tension development of the contraction curve.

Following determination of contractile properties, the regenerates were frozen in liquid nitrogen and tested histochemically for succinic dehydrogenase (13) and myosin adenosine triphosphatase (ATPase, 7, 14).

\section{RESULTS}

Contractile Properties. The development of contractile properties of noninnervated grafts (Table 1) differed substantially from that of control grafts (Table 2) which ultimately became innervated. In general, the contractile times of noninnervated grafts remained slow whereas those of innervated control grafts were slow during the early postoperative days but then progressively speeded up to resemble those of the normal extensor digitorum longus muscle. For the first 2 weeks there was little difference between the contractile characteristics of grafts placed into normal legs and those in denervated legs. By 30 days, significant differences in contractile characteristics between innervated and noninnervated grafts were already present.

An exception to this pattern was the latency period, which in both innervated and noninnervated regenerates remained relatively long. A some- 


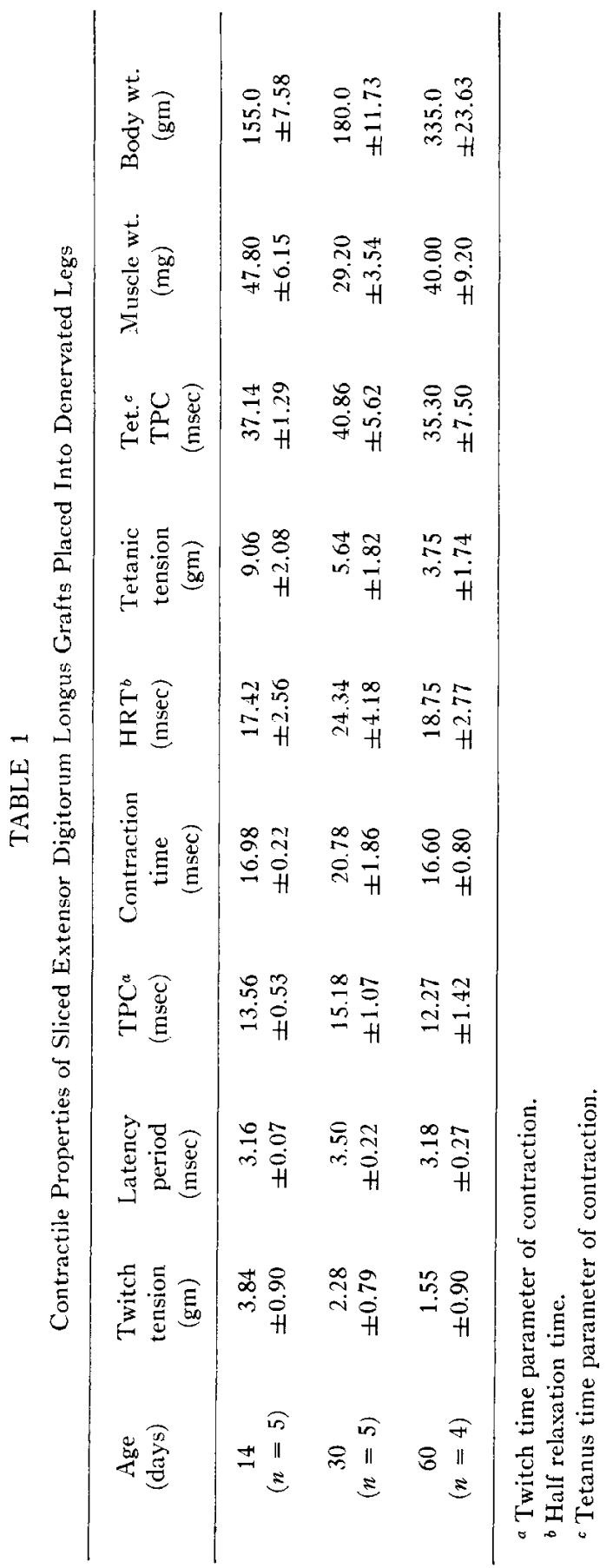


MUSCLE GRAFTS

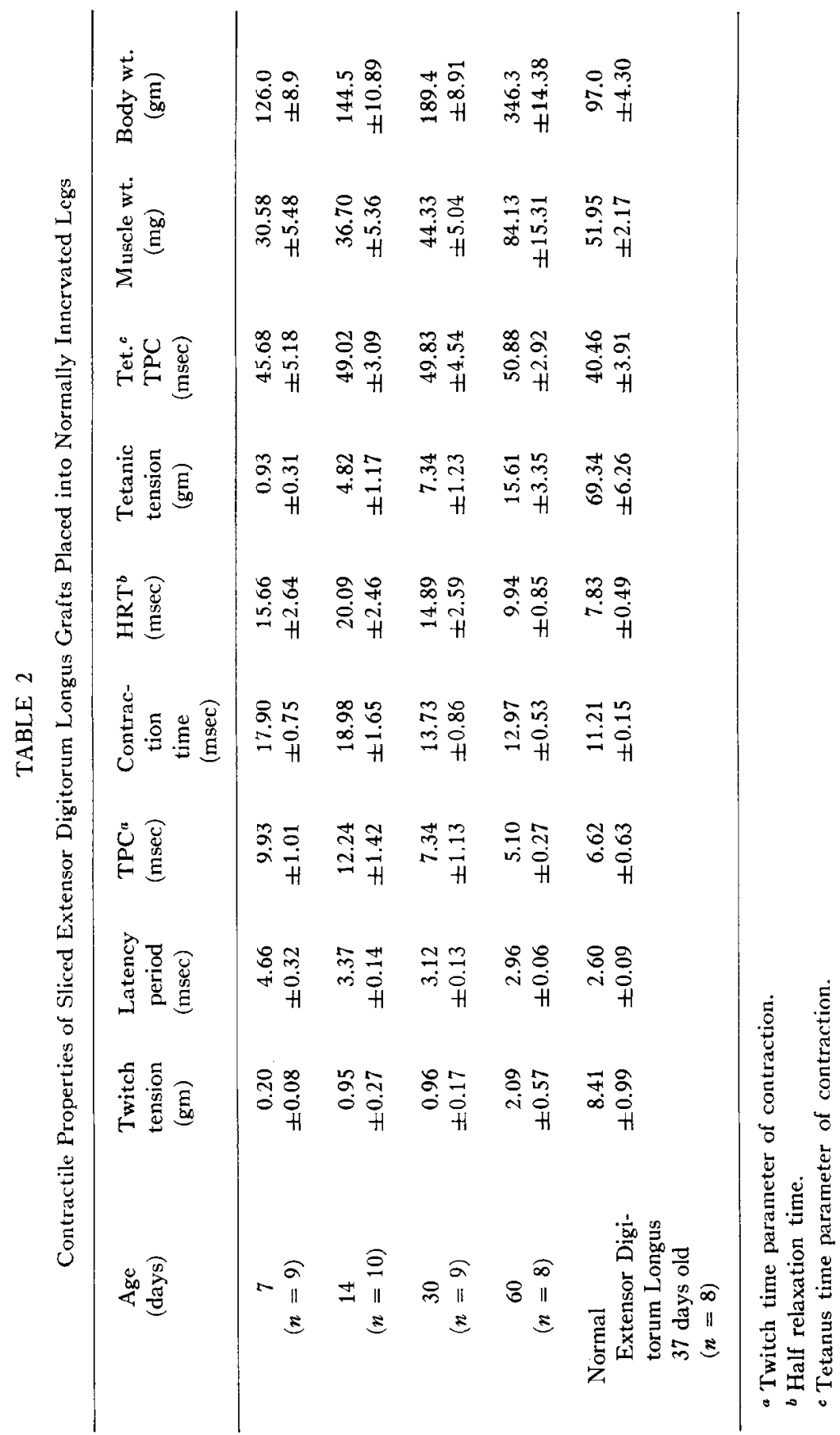


what prolunged latency period appears to be the rule in regenerated muscle (3). The contraction time of innervated grafts approached, but did not quite attain, normal speed by 60 days, the time by which contractile speeds of regenerated rat muscles have stabilized. The contraction time of 60 day noninnervated grafts, however, remained significantly more prolonged than that of either 60 day innervated grafts or the normal control extensor digitorum longus. An even more striking lack of maturation was noted in both the maximal rate of tension development (twitch time parameter of contraction) and the half relaxation time, where these times in noninnervated grafts remained about twice as long as those of both innervated grafts and normal muscle.

There was little difference in the twitch tensions generated between innervated and noninnervated grafts, but the tetanic tensions of the innervated grafts steadily rose with time, whereas those of the noninnervated grafts fell during the same period. As is usual with regenerated muscles (either minced or free grafts), the strength of contraction was considerably less (about $25 \%$ of normal) than that of the unoperated extensor digitorum longus from animals of the same size.

Histochemical Properties. During the first week after transplantation no nerves were present in free muscle grafts, even in normally innervated limbs. Within the grafts, muscle regeneration had passed through the myoblastic and myotubal stages, and at the end of the first week the myotubes were developing into immature cross-striated muscle fibers. These muscle fibers were very thin and showed a low degree of staining for both succinic dehydrogenase (Fig. 1a) and ATPase (Fig. 2a). There was no difference in the histology or histochemical activity of succinic dehydrogenase or ATPase between 7 day grafts in normal limbs and those in denervated limbs.

Thirty day muscle regenerates in normal limbs are innervated and possess motor end plates $(2,12,20)$. The muscle fibers within 30 day sliced grafts were still substantially thinner than normal muscle fibers, but in other respects they were histologically mature. In contrast to their relative structural maturity, the regenerated muscle fibers were still histochemically immature, and the mature grafts placed into normal limbs could not be distinguished from those within denervated limbs by their degree of succinic dehydrogenase or ATPase activity. All muscle fibers stained positively and homogeneously for ATPase activity, whether the grafts were in normal or denervated limbs (Fig. $2 \mathrm{~b}, \mathrm{e}$ ). No indications of distinct staining of fiber types were detected. Similarly, the succinic dehydrogenase reactions of both innervated and nerveless grafts were nonspecific and gave only the earliest indications of the emergence of histologically distinct nuscle fiber types (Fig. 1b, e). 


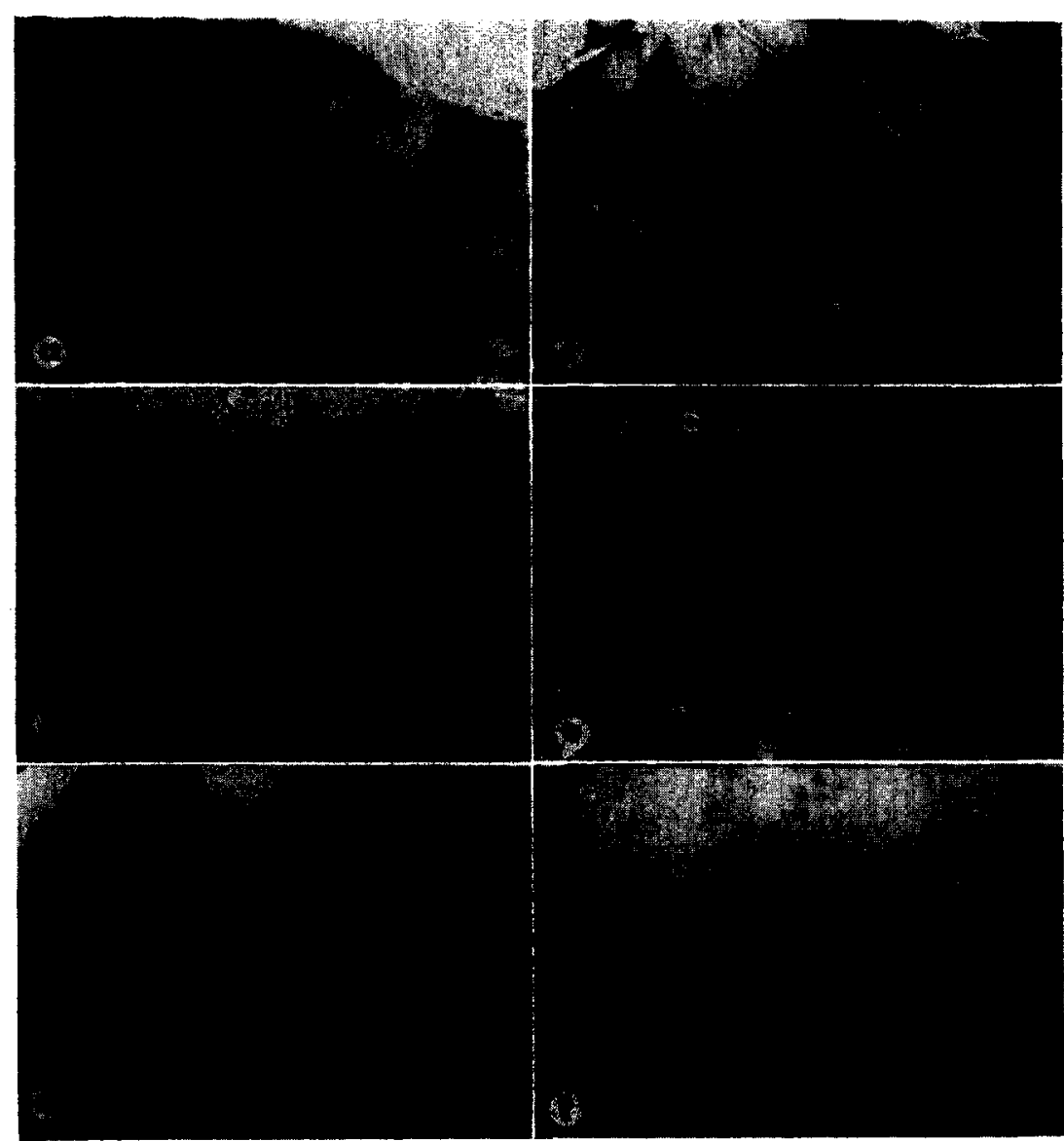

FIg. 1. Succinic dehydrogenase staining reactions of rat extensor digitorum longus sliced grafts placed in normally innervated and denervated limbs. A. 7 day regenerate in normally innervated leg. Because nerve fibers have not yet entered the graft, there is no difference between this muscle and one placed into a denervated limb. B. 30 day regenerate in normally innervated leg. C. 60 day regenerate in normally innervated leg. D. Control muscle. E. 30 day regenerate in denervated leg. F. 60 day regenerate in ienervated leg. $(\times 100)$.

By 60 days, however, grafts within normally innervated limbs stained heterogeneously (i.e., showed type I and II fibers) with respect to ATPase activity (Fig. 2c). Different fiber types could also be seen in sections stained for succinic dehydrogenase (Fig. 1c) and phosphorylase. In contrast, the muscle fibers of 60 day noninnervated grafts gave no indication of histochemical differentiation into specific types (Fig. 2f) but instead retained the pattern of histochemical homogeneity characteristic of imma- 


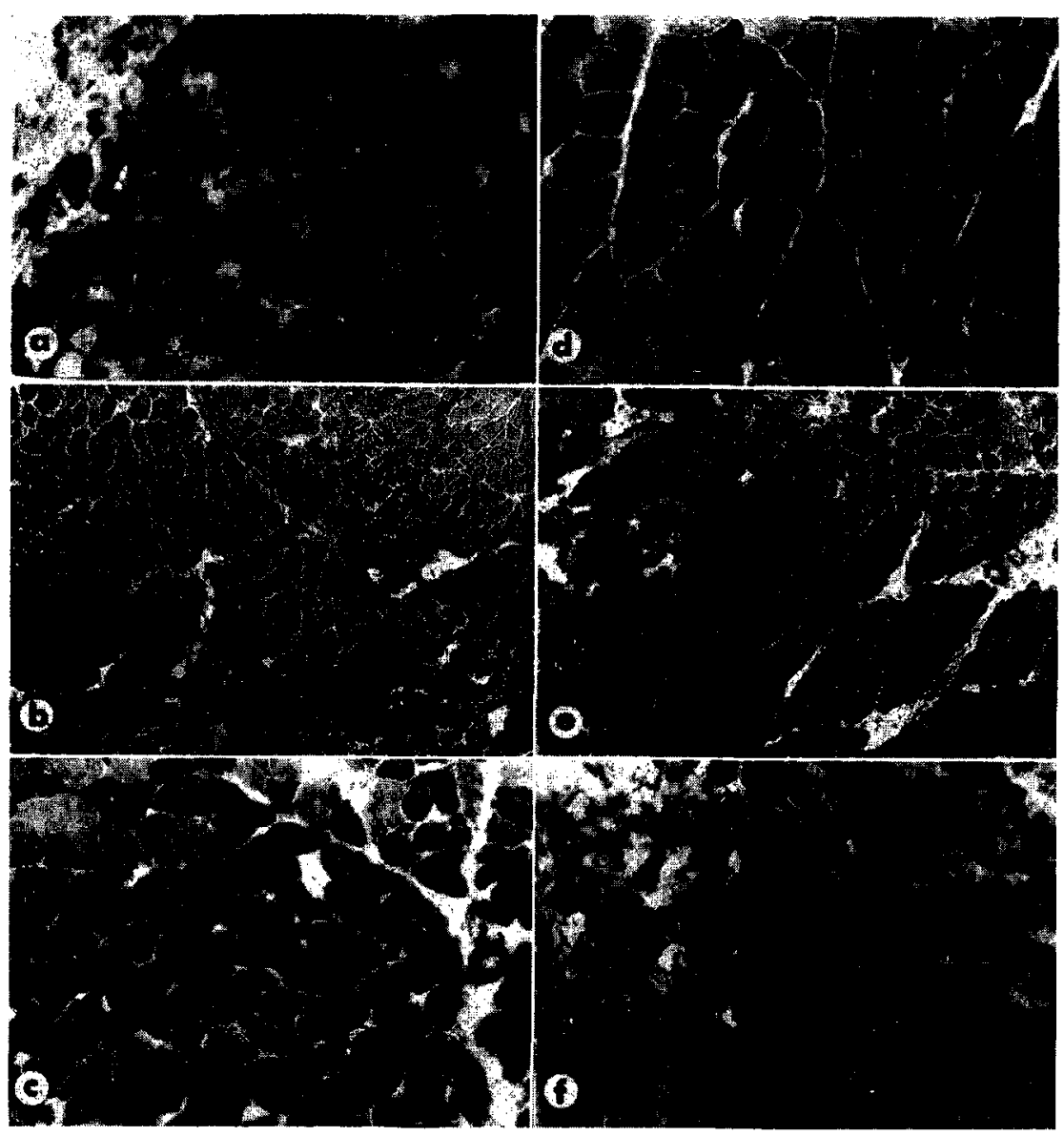

FIg. 2. ATPase staining reactions of rat extensor digitorum longus sliced grafts placed in normally innervated and denervated limbs. A. 7 day regenerate in normally innervated leg. B. 30 day regenerate in normally innervated leg. C. 60 day regenerate in normally innervated leg. D. Control muscle. E. 30 day regenerate in denervated leg. F. 60 day regenerate in denervated leg. $(\times 100)$.

ture muscle. In addition to being histochemically immature the noninnervated regenerating muscle fibers cease to grow. This can easily be seen by comparing their diameters with those of muscle fibers in innervated grafts (cf., Fig. $1 \mathrm{c}$ and f, Fig. $2 \mathrm{c}$ and $\mathrm{i}$ ). Although measurements of muscle fiber diameter were not made, it was evident from histological examination of muscle cross sections that some atrophy of the regenerating muscle fibers within the noninnervated grafts had already occurred (cf., Fig. 1e and f, Fig. 2e and f). 


\section{DISCUSSION}

By now a fairly clear picture of the ability of rat muscle to regenerate in the absence of nerves is taking shape. The absence of innervation does not impose any specific block in the differentiation of regenerating muscle fibers up to the histological stage characterized by the presence of crossstriations and peripherally migrated nuclei. In fact, for the first month it is not easy in either a minced muscle or a free graft to detect any consistent morphological differences between muscle fibers regenerating in a normally innervated limb and those in a denervated limb. The absence of major differences is due at least in part to the fact that both of these experimental models involve an early phase during which the nerve-muscle connections are necessarily interrupted. Thus regenerating nerve fibers do not normally begin to appear among the regenerating muscle fibers until the second postoperative week, and primitive motor end plates are not seen until the end of the third week $(2,11,12,20)$. Despite the lack of morphological or histochemical differences during the first month, there is a substantial difference in the contractile properties between innervated and noninnervated regenerates. Whereas 30 day innervated regenerates are making a rapid transition from slow to fast contractile properties, noninncrvated regenerates continue to contract very slowly like immature muscle. It is noteworthy that at 30 days in both the minced gastrocnemius $(3,12)$ and in the sliced extensor digitorum longus of the rat there is a pronounced shortening of contraction before clearly distinct fiber types are distinguishable with succinic dehydrogenase and ATPase staining.

Between 30 and 60 days the diameters of innervated regenerating muscle fibers increase greatly; the cuntractile properties of the muscles become stabilized at near-normal levels; and a heterogeneous pattern of histochemically distinct muscle fiber types emerges, reflecting the differential activities of the motor neurons. After reaching a low peak of differentiation at 30 days, the muscle fibers of noninnervated regenerates begin a long period of atrophy, during which the muscle fibers undergo a gradual reduction in diameter while remaining immature in their histochemical and contractile properties. The differences between innervated and noninnervated regenerates continue to increase, until by 90 days muscle fibers in noninnervated regenerates are barely recognizable whereas those of innervated regenerates have attained nearly normal dimensions (12).

It is now apparent that in the absence of nerves, regenerating muscle can differentiate to a certain level that undoubtedly represents the inherent myogenic potential for development, characterized by histological differentiation up to cross-striated muscle fibers with peripheral nuclei, slow and weak contractile properties and a lack of differentiation of the muscle fibers 
into subpopulations separable histochemically by their enzymatic activity. The presence of nerves is associated with the speeding up of contraction of a regenerating fast muscle, the enzymological differentiation of subpopulations of muscle fibers and the great increase in diameter of the muscle fibers. Because in these experimental models the noninnervated muscle is regenerating in a limb that is essentially without function, one must be cautious about attributing the final stages of differentiation of normal regenerates to the presence of nerve fibers alone. Further experimentation is necessary to determine the extent to which the final differentiation of structure, enzymatic activity and function of regenerating muscle is due to nerves alone and how much may be attributed to the functional environment in which the muscle is regenerating.

In its response to denervation as well as under normal conditions, the regeneration of rat muscle seems to recapitulate its ontogenetic development. Denervation at birth retards the structural, histochemical, and functional differentiation of muscle fibers in the rat $(6,9,18)$, although the muscles do differentiate thin striated muscle fibers. In tissue culture as well, the differentiation of histochemically distinct muscle fiber types does not occur in chick myoblasts cultured without nerves (1).

The significant ability of muscle to regenerate in the absence of nerves could be exploited to investigate differences in the inherent myogenic properties of different groups of animals. Myogenic differences due to aging are currently being investigated in our laboratories. This model of regenerating nerveless muscle could be of use in investigating differences between normal and diseased (for example dystrophic) muscle to ascertain whether or not there are any inherent differences not directly dependent upon an intact nerve supply.

\section{REFERENCES}

1. Askanas, V., S. A. ShafiQ, and A. T. Milhorat. 1972. Histochemistry of cultured aneural chick muscle. Morphological maturation without differentiation of fiber types. Exp. Neurol. $37: 218-230$.

2. Bennett, M. R., T. J. Florin, and R. H. Woog. 1974. The formation of synapses in regenerating mammalian striated muscle. J. Physiol. (London) 238: 79-92.

3. Carlson, B. M., and E. Gutmann. 1972. Development of contractile properties of minced muscle regencrates in the rat. Exp. Ncurol. 36: 239-249.

4. Carlson, B. M., and E. Gutmann. 1975. Regeneration in grafts of normal and denervated rat muscles. Contractile properties. Pfïgers Arch. 353: 215-225.

5. Carlson, B. M., and E. Gutmann. 1975. Regeneration in free grafts of normal and denervated muscles in the rat: Morphology and histochemistry. Anat. Rec. $183: 47-62$.

6. Engel, W. K., and G. KARPATi. 1968. Impaired skeletal muscle maturation following neonatal neurectomy. Dcvel. Biol. $17: 713-723$.

7. Guth, L., and F. J. Samaha. 1970. Procedure for the histochemical demonstration of actomyosin ATPase. Exp. Neurol. 28: 365-367. 
8. Gutmann, E., and B. M. Carlson. 1975. A comparison between the free grafting of sliced and intact muscles in the rat. Experientia. 31: 848-849.

9. Hanzlikova, V., and S. Schiaffino. 1973. Studies on the effect of denervation in developing muscle. III. Diversification of myofibrillar structure and origin of the heterogeneity of muscle fiber type. Z. Zellforsch. 147:75-85.

10. Hsu, L. 1974. The role of nerves in the regeneration of minced skeletal muscle in adult anurans. Anat. Rec. $179: 119-136$.

11. Mong, F. S. F. 1975. Nervous influences on minced muscle regeneration in rats (Abstr.). Anat. Rec. $181: 429$.

12. Mong, F. S. F. 1975. Histological and histochemical studies on the nervous influence on minced muscle regeneration of the triceps nerve of the rat. Ph.D. Thesis, University of Michigan, Ann Arbor.

13. Nachlas, M. K., K. C. Tsou, E. Souza, C. S. Cheng, and A. M. Seligman. 1957. Cytochemical demonstration of succinic dehydrogenase by the use of a new P-nitrophenyl substituted ditetrazole. J. Histochem. Cytochem. 5 : 420-436.

14. Padykula, H. A., and E. Herman. 1955. The specificity of the histochemical method for adenosine triphosphate. J. Histochem. Cytochem. 3: 170-195.

15. RohličEK, V. 1968. An automatic analyzer of muscle contraction. SNTL Tech. Dig. 61 : 383-387.

16. RohličeK, V., and E. GutMans. 1972. Time constant of contraction, a new expression of maximal rate of tension development. Physiol. bohemoslov. 21 : 430-431.

17. Salarsky, B. 1971. Functional studies of regenerated muscles from normal and dystrophic mice. Nature (London) $229: 270-273$.

18. Shafiq, S. A., S. A. Asiedu, and A. T. Milhorat. 1972. Effect of neonatal neurectomy on differentiation of fiber types in rat skeletal muscle. Exp. Neurol. 35 : $529-540$.

19. Studitsky, A., R. Zhenevskaya, and O. Rumyantseva. 1963. The role of neurotrophic influences upon the restitution of structure and function of regenerating muscles, pp. 71-82. In "The Effects of Use and Disuse on Neuromuscular Functions." E. Gutmann and P. Hnik [Eds.]. Publ. House of Czechoslovak Acad. Sci., Prague.

20. Zhenevskaya, R. P. 1962. Experimental histologic investigation of striated muscle tissue. Rev. Canad. Biol. 21 : 457-470. 